\title{
Rawdata-Based Detection of the Optimal Reconstruction Phase in ECG-Gated Cardiac Image Reconstruction
}

\author{
Dirk Ertel $^{1}$, Marc Kachelrie ${ }^{1}$, Tobias Pflederer ${ }^{2}$, Stephan Achenbach ${ }^{2}$, \\ Robert M. Lapp ${ }^{3}$, Markus Nagel ${ }^{1}$, and Willi A. Kalender ${ }^{1}$ \\ ${ }^{1}$ Institute of Medical Physics, University of Erlangen-Nürnberg, Germany \\ dirk. ertel@imp. uni-erlangen. de \\ ${ }^{2}$ Department of Internal Medicine II, University of Erlangen-Nürnberg, Germany \\ ${ }^{3}$ VAMP GmbH, Erlangen, Germany
}

\begin{abstract}
In order to achieve diagnostically useful CT (computed tomography) images of the moving heart, the standard image reconstruction has to be modified to a phase-correlated reconstruction, which considers the motion phase of the heart and generates a quasi-static image in one defined motion phase. For that purpose a synchronization signal is needed, typically a concurrent ECG recording. Commonly, the reconstruction phase is adapted by the user to the patient-specific heart motion to improve the image quality and thus the diagnostic value. The purpose of our work is to automatically identify the optimal reconstruction phase for cardiac CT imaging with respect to motion artifacts. We provide a solution for a patient- and heart rate-independent detection of the optimal phase in the cardiac cycle which shows a minimum of cardiac movement. We validated our method by the correlation with the reconstruction phase selected visually on the basis of ECG-triggering and used for the medical diagnosis. The mean difference between both reconstruction phases was $12.5 \%$ with respect to a whole cardiac motion cycle indicating a high correlation. Additionally, reconstructed cardiac images are shown which confirm the results expressed by the correlation measurement and in some cases even indicating an improvement using the proposed method.
\end{abstract}

\section{Introduction}

In general, the possibility of imaging moving objects in a quasi-static way is strongly dependent on the temporal resolution of the imaging system. In the special case of cardiac CT, the limited temporal resolution leads to motion artifacts when using standard image reconstruction algorithms (cp. Figure 1). Under the assumption of a quasiperiodic motion of the object, the reconstruction algorithm can be synchronized with the object's movement [1 $\left[\begin{array}{lll}1 & 3 & 4\end{array}\right]$. Here, only projection data of the same motion phase are used for the image reconstruction, while the projection data acquired during other phases do not contribute to the image [2]. Periodic recurrent states indicated as synchronization points are detected. With respect to these synchronization points, a phase is defined which indicates the motion phase in which the heart is being imaged. In a temporal window around the defined phase, the projection data are used for the image reconstruction. In this way, the temporal resolution can be increased artificially and the object can be imaged in a quasi-static way. For cardiac imaging usually the ECG is used 


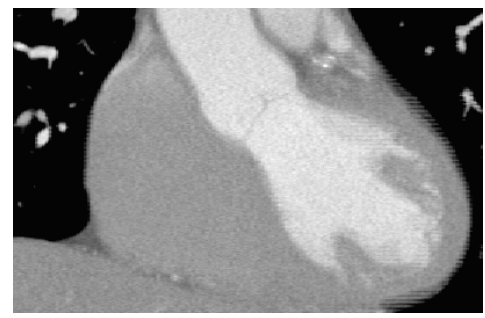

a)

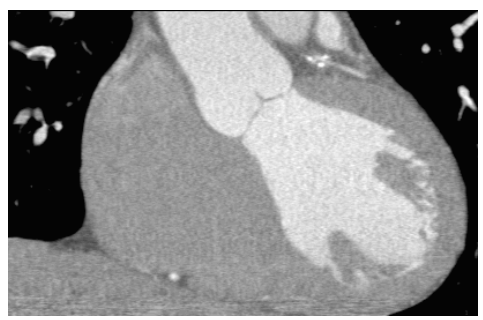

b)

Fig. 1. Example of cardiac images using standard reconstruction (a) and phase-correlated reconstruction (b)

as a synchronization function representing the cardiac cycle, i.e. the periodic contraction [2]. As an alternative synchronization signal, the kymogram function can be used (cp. Figure 2 (a)). It is generated by the temporal variation of the mass [5,67]. Due to the cardiac contraction, the mass of the projected slices varies with time and correlates with the heart rate. Thus, the kymogram can be computed without additional devices, directly from the CT rawdata. In contrast to the ECG, it captures the true mechanical motion of the heart and not an indirect signal like the ECG.

Since the heart's contractile motion is a non-uniform and only quasi-periodic motion, not all phase intervals in the cardiac cycle are equally suited with respect to the phasecorrelated reconstruction algorithm. The systolic phase corresponds to the contraction of the heart and the myocardium shows a high velocity. After the following relaxation the heart is at rest for a moment which corresponds to the diastolic phase. The diastolic phase represents the optimal cardiac reconstruction phase for most cases [8], since the temporal window for the data acquisition covers the complete period of rest. With increasing heart rate, the systolic phase more frequently provides optimal image quality [9]. However, a general prediction of the optimal reconstruction phase showing a minimum of motion is not possible. On the one hand, the possibility of a reconstruction in the systolic or the diastolic phase prohibits this prediction. On the other hand, a slight patient-dependent variation of the typical ECG phases makes the prediction uncertain. Hence, it is common practice to approach the optimal image quality adaptively by iterative image reconstruction at different phase points. An image-based method to detect the optimal reconstruction phase already exists [10]. There, the optimal reconstruction phase can be computed by a similarity measure of the image stacks reconstructed at different phase points leading to the best achievable image quality. Both approaches, the user-based iterative way and the automatic image-based detection, require multiple image stacks and therefore a large number of image reconstructions have to be performed. Thus, they are very time consuming and computationally expensive. Moreover, all image stacks have to be visually assessed for the user-based approach.

We propose a fully automatic computer-assisted detection of the optimal reconstruction phase using the rawdata-based kymogram signal. Our approach intends to provide optimal image quality without the need for performing more than the final image reconstruction. This may allow substantial reductions in the time necessary for processing cardiac CT data sets in the medical workflow. 


\section{Materials and Methods}

The proposed method detects the optimal phase for a phase-correlated cardiac image reconstruction using the rawdata-based kymogram signal [67] which represents the heart's motion function. The kymogram signal is generated by the standard algorithm from Kachelrieß et al. [67]. The algorithm computes the center-of-mass (COM) point of the projected slice which varies over time due to the heart's motion. The twodimensional kymogram signal

$$
\boldsymbol{r}_{\mathrm{c}}(t)=\left(\begin{array}{l}
x_{\mathrm{c}}(t) \\
y_{\mathrm{c}}(t)
\end{array}\right)
$$

represents the motion of the COM-point $\boldsymbol{r}_{\mathrm{c}}$ of a transversal slice in the direction of $x$ and $y$ with time $t$. For a detailed description of the quasi-periodic heart motion we use the motion phase $p$. It indicates the relative point in time in one period of the moving heart by a normalization to the length of the period $T$. For covering the whole scan time $t \in\left[t_{\text {Start }}, t_{\text {End }}\right]$ we obtain the modular phase $p(t) \in[0,1]$

$$
p(t)=\frac{t-t_{n}}{T_{n}} .
$$

Since we cover more than one period we have to assume a variable heart rate $1 / T_{n}=$ $1 /\left(t_{n+1}-t_{n}\right)$ with the synchronization points $t_{n}, t_{n+1}$ and $t_{n} \leq t<t_{n+1}$. Figure 2 (b) shows an example of the modular phase $p(t)$ and the corresponding ECG signal where the R-peaks are defined as the synchronization points $t_{n}$. A grey-coded twodimensional plot of $\boldsymbol{r}_{\mathrm{c}}(t)$ is shown in Figure 3 (a). The grey-coding is defined with respect to the current heart motion phase $p(t)$ shown in Figure 2 (b). The grey-coded scatterplot in Figure 3 (a) already shows a correlation between the location of the COM points and the heart motion phases as was indicated in [6]. The light-grey points corresponding to the systolic phase $p(t)=20 \%-40 \%$ are mainly located in the left bottom corner of Figure 3 (a). The dark points corresponding to the diastolic phase $p(t)=60 \%-80 \%$ are mainly located in the middle of the scatterplot. Since the heart's motion function is a quasi-periodic function in the following we are just interested in the patient-specific phase motion function $\boldsymbol{r}_{\mathrm{c}}(p)$ of a heart's motion cycle with respect to the motion phase $p$. For that purpose, we observe each motion cycle with length $T_{n}$ separately within the continuous time signal $\boldsymbol{r}_{\mathrm{c}}(t)$. We obtain the phase motion function $\boldsymbol{r}_{\mathrm{c}}(p)$ by averaging the heart's motion function $\boldsymbol{r}_{\mathrm{c}}(t)$ over the whole scan time with respect to the phase $p$. By using a modified histogram function we can extract all dedicated values of $\boldsymbol{r}_{\mathrm{c}}(t)$ for the phase $p$ and compute the mean value

$$
\boldsymbol{r}_{\mathrm{c}}(p)=\frac{1}{\int_{t_{\mathrm{Start}}}^{t_{\mathrm{End}}} \delta(p(t)-p) d t} \int_{t_{\mathrm{Start}}}^{t_{\mathrm{End}}} \delta(p(t)-p) \cdot \boldsymbol{r}_{\mathrm{c}}(t) d t .
$$

Thus, we generate a representative motion function of the COM points for a heart's motion cycle. An example of a phase motion function $\boldsymbol{r}_{\mathrm{c}}(p)$ of a chosen patient is shown in Figure 3 (b). The velocity of the heart with respect to the motion phase can be identified by the distance between two neighboring phase-based COM points $\boldsymbol{r}_{\mathrm{c}}(p)$ 
a)
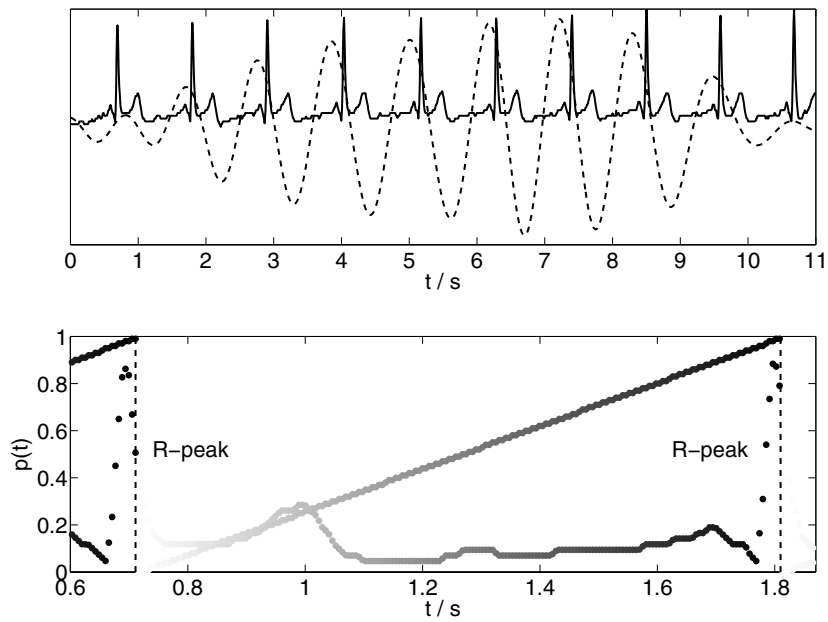

b)

Fig. 2. ECG-signal (solid) and corresponding kymogram function (dashed) (a); RR-cycle of an ECG-signal and corresponding modular phase $p(t)$ (b)

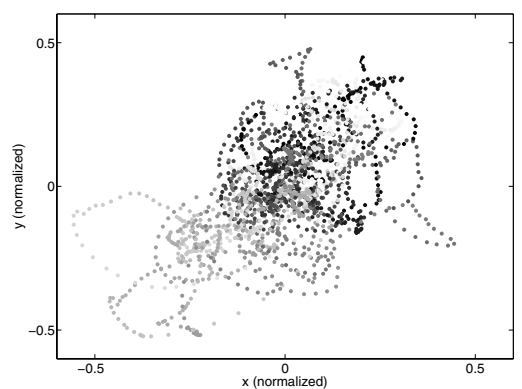

a)

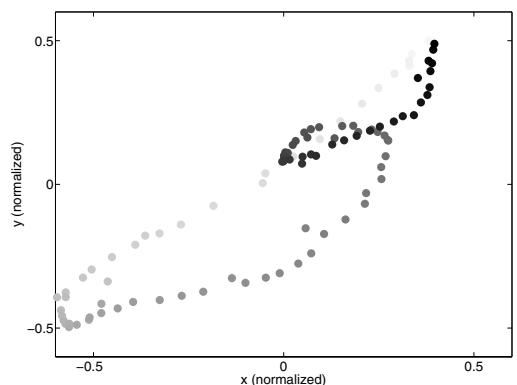

b)

Fig. 3. Example (a) of a motion function $\boldsymbol{r}_{\mathrm{c}}(t)$ over the whole scan time and (b) of a phase motion function $\boldsymbol{r}_{\mathrm{c}}(p)$ of a chosen patient

and $\boldsymbol{r}_{\mathrm{c}}(p+\Delta p)$. For a constant phase-based sampling rate $\Delta p$ two neighboring COM points have a higher distance $\left|\boldsymbol{r}_{\mathrm{c}}(p+\Delta p)-\boldsymbol{r}_{\mathrm{c}}(p)\right|$ for a higher velocity than for lower velocity. As already explained in the introduction projection data from a temporal window around the selected phase are used for the image reconstruction. Hence, we detect the optimal reconstruction phase $p_{\text {algo }}$ by identifying the phase point with the lowest mean velocity in the considered period (temporal window). Therefore, we minimize the distance of a phase-based COM point $\boldsymbol{r}_{\mathrm{c}}(p)$ with respect to the selected phase $p$ to the neighboring points $\boldsymbol{r}_{\mathrm{c}}\left(p+p_{\mathrm{i}}\right)$ in a relevant range $2 \cdot p_{\mathrm{w}}$ 


$$
\left.p_{\text {algo }}=\operatorname{argmin}_{p}\left(\int_{-p_{\mathrm{w}}}^{p_{\mathrm{w}}} \| \boldsymbol{r}_{\mathrm{c}}\left(p+p_{\mathrm{i}}\right)-\boldsymbol{r}_{\mathrm{c}}(p)\right) \| d p_{\mathrm{i}}\right) .
$$

This relevant range is defined by the relative temporal resolution of the phase-correlated cardiac reconstruction algorithm [3[11], contributing to the image reconstruction.

For validation, we used data of 65 consecutive patients scanned with standard protocols, a collimation of $2 \cdot 32 \times 0.6 \mathrm{~mm}$, a rotation time of $0.33 \mathrm{~s}$ and a concurrent ECG recording (Sensation 64 Siemens Medical Solutions, Forchheim, Germany). For all patients, a phase-correlated image reconstruction had already been performed with the reconstruction phase determined iteratively by a cardiologist using visual assessment to identify the optimal cardiac phase. We assume that this reconstruction phase leads to images holding optimal quality. We defined the quality measure $Q$ as the absolute difference of the identified optimal reconstruction phase $p_{\text {algo }}$ and the reconstruction phase used for the medical diagnosis $p_{\text {med }}$

$$
Q=\left\{\begin{array}{cl}
\left|p_{\text {algo }}-p_{\text {med }}\right| & \text {, for }\left|p_{\text {algo }}-p_{\text {med }}\right| \leq 50 \% \\
100 \%-\left|p_{\text {algo }}-p_{\text {med }}\right| & \text {, for }\left|p_{\text {algo }}-p_{\text {med }}\right|>50 \%
\end{array} .\right.
$$

\section{Results}

The mean value of the quality measure over all patients was $\bar{Q}_{\mathrm{I}}=13.9 \%$ with a standard deviation of $\sigma=11.1 \%$. For 4 patients the kymogram-based algorithm proposed the systolic phase as the optimal reconstruction phase while the diastolic phase had been chosen by the medical expert for the medical diagnosis. In detail, our algorithm predicted a reconstruction phase between $23 \%$ and $41 \%$ while a reconstruction phase of $65 \%$ or $70 \%$ had been determined by the cardiologist. The 4 patients degrade the quality measure strongly whereas the diagnostic relevance remains to be proven. After excluding these patients, the mean difference decreases to $\bar{Q}_{\mathrm{II}}=12.5 \%$ with a standard deviation of $\sigma=9.8 \%$. Figure 4 shows two histogram functions of both quality measures $\bar{Q}_{\mathrm{II}}$ and $\bar{Q}_{\mathrm{I}}$.

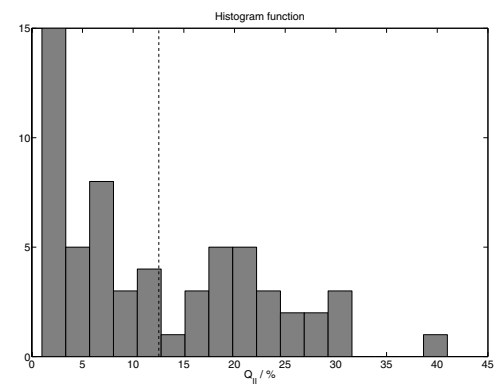

a)

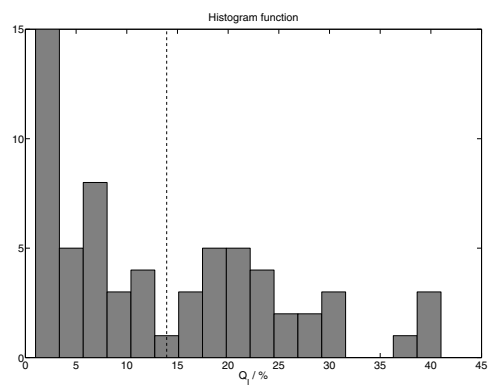

b)

Fig. 4. Histogram function of the quality measure $Q$ from (a) the selected 61 patients and (b) all consecutive 65 patients including the mean values (dashed line) 

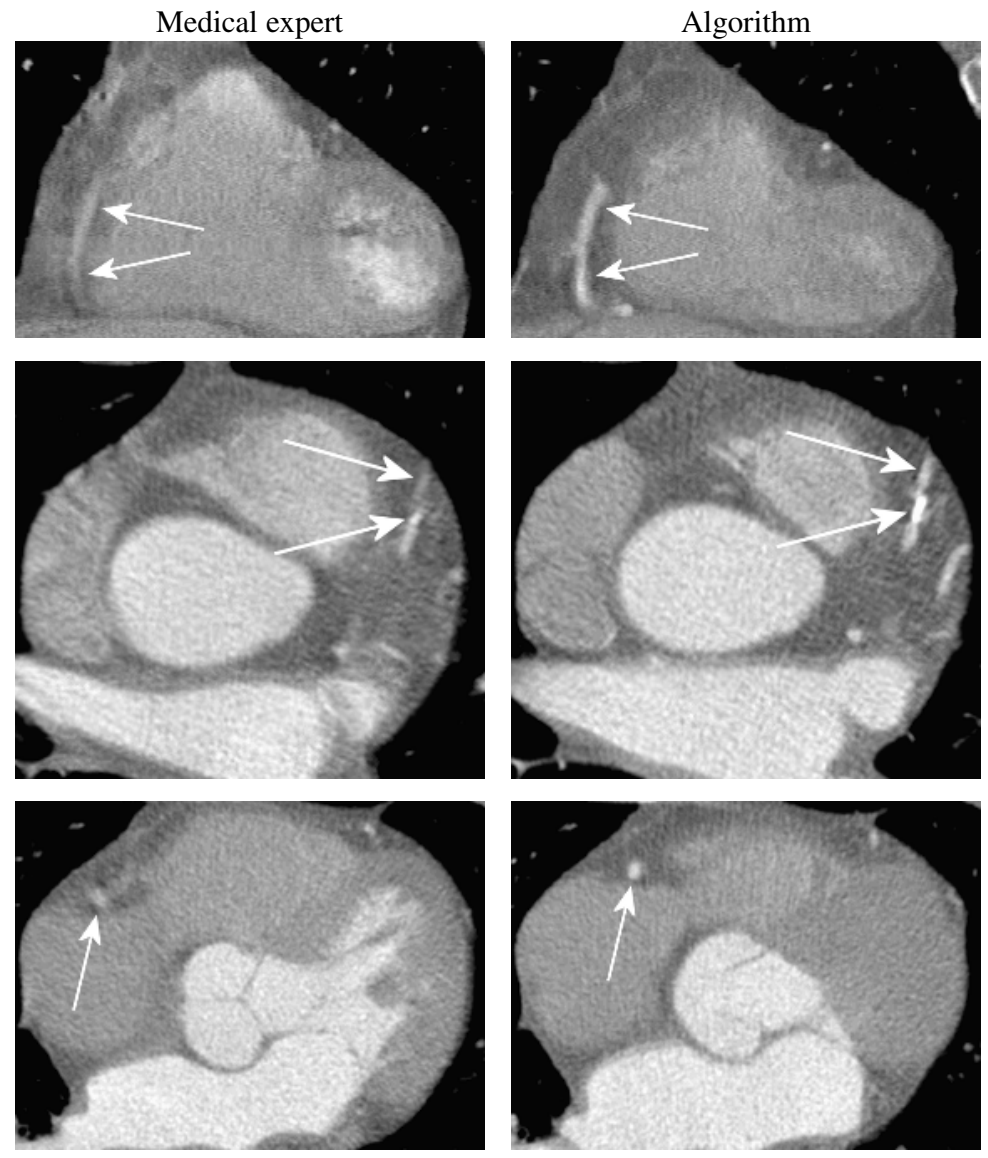

Fig. 5. Example 1: Phase-correlated cardiac images $\left(f_{\mathrm{H}}=75.1 \mathrm{bpm}\right)$ in coronal view (top) and two different transaxial views (middle and bottom) with $p_{\text {med }}=70 \%$ (left) and $p_{\text {algo }}=29 \%$ (right) (C 0/W 1000)

Figure 5 serves as an example to illustrate our decision to exclude the predicted systolic phases from the objective quality measure when the diastolic phase had been used by the cardiologist. For this patient $p_{\text {algo }}=29 \%$ was predicted and $p_{\text {med }}=70 \%$ had been used. One coronal view and two transaxial slices are shown for both reconstruction phases. The images reconstructed at $p_{\text {med }}$ suffer from motion artifacts (right images). The left circumflex (LCX) artery shown in the coronal view (top) and the left anterior descending (LAD) artery shown in the first transaxial slice (middle) are strongly blurred. The right coronary artery (RCA) shown in the second transaxial slice (bottom) has no sharp delineation between the vessel and the surrounding structures which is a typical motion artifact. A reconstruction at $p_{\text {algo }}$ (left images) clearly improves the image quality. Blurring of the LCX and the LAD is reduced and the RCA shows a sharper delineation. Also the calcification in the LAD is more visible. 

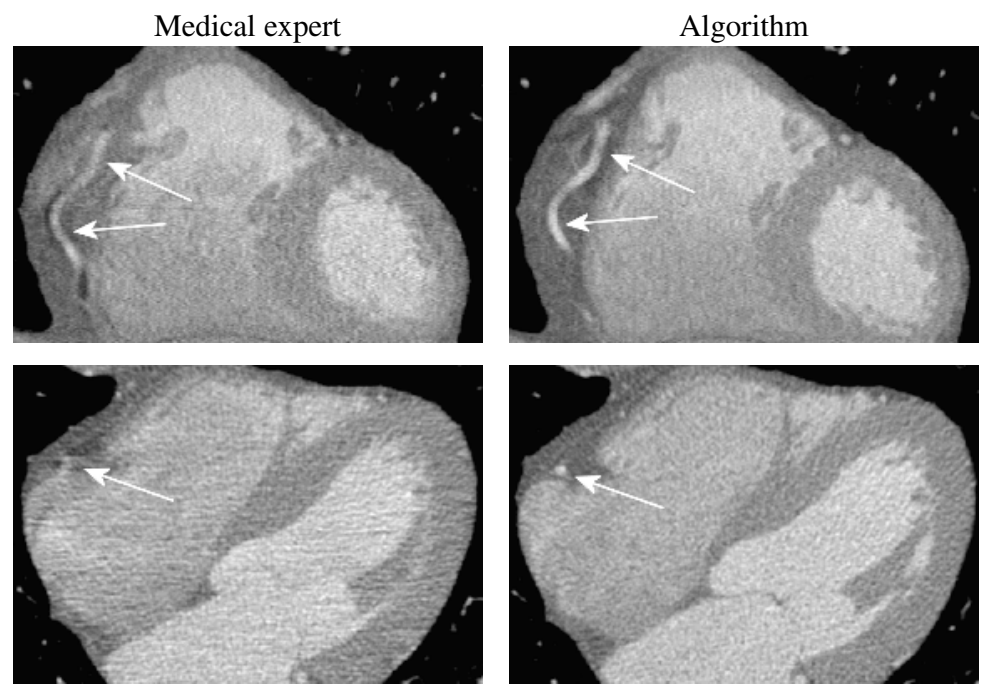

Fig. 6. Example 2: Phase-correlated cardiac images $\left(f_{\mathrm{H}}=63.8 \mathrm{bpm}\right)$ in coronal view (top) and transaxial view (bottom) with $p_{\text {med }}=60 \%$ (left) and $p_{\text {algo }}=76 \%$ (right) $(\mathrm{C} \mathrm{0/W} \mathrm{1000)}$

Moreover, Figure 6 shows an example with a higher-than-average deviation between the proposed phase $p_{\text {algo }}=76 \%$ (left images) and the used phase $p_{\text {med }}=60 \%$ (right images) and also with increasing image quality. Again, a coronal view and a transaxial slice are shown for both reconstruction phases. The LCX shown in the coronal view (top) is blurred and the RCA shown in the transaxial slice (bottom) has no sharp delineation. Both effects are reduced for a reconstruction at $p_{\text {algo }}$. Using the proposed casespecific optimal reconstruction phase in diastole, an improvement in image quality was achieved.

\section{Discussion}

We presented a kymogram-based approach to automatically identify the optimal reconstruction phase and thereby obtain motion-free cardiac CT images. The proposed method was implemented on a standard PC with a computation time of under 2 minutes per patient and can be easily made usable on medical workstations. Multiple timeconsuming image reconstructions and other proposed image-based methods may become obsolete. We achieved good correlation of the predicted reconstruction phase with the reconstruction phase chosen by the medical expert. The mean difference value of both reconstruction phases was $\bar{Q}_{\mathrm{I}}=13.9 \%$ and $\bar{Q}_{\mathrm{II}}=12.5 \%$, respectively. Moreover, in two of several examples we have shown that the image quality can be even improved using the predicted reconstruction phase compared to the phase used for the medical diagnosis which was chosen empirically. We conclude that the presented kymogrambased method can improve the medical workflow by providing optimal image quality with the initial image reconstruction. Second it provides optimal image quality by detecting the optimal reconstruction phase which is dependent on the heart rate and also 
on the patient. Since empirically chosen reconstruction phases provide suboptimal image quality in some cases, our computed-assisted method has the potential to improve image quality.

\section{References}

1. DeMan, B., Edic, P., Basu, S.: An iterative algorithm for time-solved reconstruction of a CT scan of a beating heart. (2005) 356-359 The Eighth International Meeting on Fully Three-dimensional Image Reconstruction in Radiology and Nuclear Medicine.

2. Kachelrieß, M., Kalender, W.A.: Electrocardiogram-correlated image reconstruction from subsecond spiral computed tomography scans of the heart. Medical Physics 25 (1998) 24172431

3. Kachelrieß, M., Ulzheimer, S., Kalender, W.A.: ECG-correlated image reconstruction from subsecond multi-slice spiral CT scans of the heart. Medical Physics 27 (2000) 1881-1902

4. Kubo, H., Hill, B.: Respiratory gated radiotherapy treatment: A technical study. Physics in Medicine and Biology 41 (1996) 83-91

5. Bruder, H., Maguet, E., Stierstorfer, K., Flohr, T.: Cardiac spiral imaging in Computed Tomography without ECG using complementary projections for motion detection. Proc. SPIE (2003)

6. Kachelrieß, M., Sennst, D.A., Maxlmoser, W., Kalender, W.A.: Kymogram detection and kymogram-correlated image reconstruction from subsecond spiral computed tomography scans of the heart. Medical Physics 29 (2002)

7. Kalender, W.A., Kachelrieß, M.: Computertomograph mit objektbezogener Bewegungsartefaktreduktion und Extraktion der Objektbewegungsinformation (Kymogramm) (1999) European Patent Office, Patent pending, Patent Nr.: 99111708.6 - 1522.

8. Achenbach, S., Ropers, D., Holle, J., Muschiol, G., Daniel, W.G., Moshage, W.: In-Plane Coronary Arterial Motion Velocity: Measurement with Electron-Beam CT. Radiology 216 (2000) 457-463

9. Shanneik, K., Kachelrieß, M., Kalender, W.A.: Heart Rate Dependency of Image Quality Using Multi-segment Reconstruction Algorithms. In: Supplement to Radiology, Radiological Society of North America (2005) 479 RSNA 2005 conference proceedings.

10. Manzke, R., Köhler, T., Nielsen, T., Hawkes, D., Grass, M.: Automatic phase determination for retrospectively gated cardiac CT. Medical Physics 31 (2004)

11. Kachelrieß, M., Knaup, M., Kalender, W.A.: Phase-correlated imaging from multi-threaded spiral cone-beam CT scans of the heart. (2005) 159-162 Proceedings of the 8th International Meeting on Fully 3D Image Reconstruction. 